cond-mat/9406002

\title{
Measuring the Relative Phase of the Energy Gap in a High-Temperature Superconductor
}

\author{
with EELS \\ Michael E. Flatté \\ Division of Applied Sciences, Harvard University, Cambridge, Massachusetts 02138
}

\begin{abstract}
A method of measuring the relative phase of the energy gap in a high-temperature superconductor is suggested for electron energy loss spectroscopy. Energy-resolved measurements of off-specular scattering should show a feature similar to the specular feature associated with the gap. Unlike the specular feature, which reflects an average of the gap over the (normal) Fermi surface, the energy loss of the off-specular feature depends on the superconducting energy gap at only two locations on the Fermi surface. The onset of the feature reflects the relative phase between these two points. This result is independent of surface characteristics. Such characteristics affect the magnitude of the off-specular feature, not its location or onset. The size of the feature is estimated for a simple surface model. Implications of specific measurements on $\mathrm{Bi}_{2} \mathrm{Sr}_{2} \mathrm{CaCu}_{2} \mathrm{O}_{8}$ are discussed.
\end{abstract}


The details of the energy-gap anisotropy in the high-temperature superconductors remain a critical unresolved issue. Despite recent advances in experiment, yielding strong evidence for a finite density of states at low energy [1] and angular anisotropy in the gap [2, 3], whether the gap has nodes or changes sign is not known.

This Communication will suggest that the gap magnitude and relative phase around the Fermi surface may be measured with electron-energy-loss-spectroscopy (EELS). The proposal is related to a method proposed earlier using inelastic neutron scattering measurements of phonon linewidths [4,5]. The angle-resolved influence of the gap on the phonon linewidth occurs due to the single-electron-single-hole decay channel of the phonon. The neutron-scattering experiment requires accurate measurements of the difference in phonon linewidths between the normal and the superconducting state. The small normal-state electronic linewidths in the high-temperature superconductors $\mathrm{La}_{1.85} \mathrm{Sr}_{0.15} \mathrm{CuO}_{4}$ [6], $\mathrm{YBa}_{2} \mathrm{Cu}_{3} \mathrm{O}_{7}$ [7], and $\mathrm{Bi}_{2} \mathrm{Sr}_{2} \mathrm{CaCu}_{2} \mathrm{O}_{8}$ [8] render these measurements quite difficult.

Angle-resolved off-specular electron-energy-loss spectra are sensitive to single-electronhole-pair scattering processes, and thus to the angle-resolved gap magnitude and phase. On-specular EELS has been successfully performed on $\mathrm{Bi}_{2} \mathrm{Sr}_{2} \mathrm{CaCu}_{2} \mathrm{O}_{8}$ [9], but these nearzero-momentum scattering processes yield features which depend on an average of the gap. Sharp features should appear in the loss spectra for momentum transfers greater than an inverse superconducting coherence length even if the gap is anisotropic. These features depend on the angle-resolved gap magnitude and phase at points on the Fermi surface which differ for each momentum transfer.

Simply from kinematics, an electron scattering by parallel crystal momentum $\mathbf{Q}$ with energy loss $\omega$ in a single scattering event can only [10] be affected by the superconducting electrons through the imaginary part of the polarization, $\operatorname{Im} P_{b u l k}^{r e t}(\mathbf{Q}, \omega)$. The subscript bulk indicates the polarization is calculated for an infinite, nonterminated crystal. The surface normal is arranged parallel to the trivial electronic direction $(\hat{z})$. Due to the two-dimensional electronic structure of the superconductor, no momentum variable in the $\hat{z}$ direction is required for $P_{b u l k}^{r e t}(\mathbf{Q}, \omega)$. The details of the surface will affect the magnitude of the feature 
in the scattering probability which originates from $P_{b u l k}^{r e t}(\mathbf{Q}, \omega)$.

Now the structure of $P_{b u l k}^{r e t}(\mathbf{Q}, \omega)$ for $Q>2 \pi \xi^{-1}$, where $\xi$ is the superconducting coherence length, will be discussed. The discussion is more detailed in Refs. [4.5]. Only the first-order polarization diagram produces sharp features, so only that diagram, a two-quasiparticle bubble, will be considered here. In the superconductor there is a minimum energy to create two electronic quasiparticles due to the gap. In an isotropic superconductor that minimum energy is just twice the energy gap. For a loss $\omega$ less than twice the gap $P_{b u l k}^{r e t}(\mathbf{Q}, \omega)$ is purely real; there is no scattering with loss $\omega$ due to the superconducting electrons. For $\omega$ greater than twice the gap there are electronic scattering processes. The transition between the two in $\operatorname{Im} P_{\text {bulk }}^{r e t}(\mathbf{Q}, \omega)$ is discontinuous, as predicted [11,12] and seen [13, 14, in the lowtemperature superconductors $\mathrm{Nb}_{3} \mathrm{Sn}$ and niobium in phonon linewidths (which also measure $\left.\operatorname{Im} P_{b u l k}^{r e t}(\mathbf{Q}, \omega)\right)$.

In a material with a quasi-two-dimensional Fermi surface, the two quasiparticles can only be created at certain locations on the Fermi surface, as shown in Fig. 1 (the $\mathrm{Bi}_{2} \mathrm{Sr}_{2} \mathrm{CaCu}_{2} \mathrm{O}_{8}$ Fermi surface [15]). Both quasiparticles of a pair must be created very near the Fermi surface. This is due to the large electronic energy scale of the Fermi surface (eV) compared with the losses under consideration of the scattering electrons (meV). Thus the minimum $\operatorname{loss} \tilde{\Delta}(\mathbf{Q})$ for an electronic scattering event with momentum transfer $\mathbf{Q}$ would depend on the sum of the gap magnitudes at the two points where quasiparticles are created. Those points are determined by placing the momentum transfer on the Fermi sea such that both the head and the tail lie on the Fermi surface. The quasiparticles are created at the head of the vector and the Fermi surface point opposite the tail. $\tilde{\Delta}(\mathbf{Q})$ is frequently multivalued. As shown in Fig. 1, for a momentum $0.5(\pi, \pi)$, there are three possible pairs of quasiparticles (labeled $1-3$ on Fig. 1). Each of these three thresholds may be independently observed as a discontinuity in the differential scattering of electrons.

$Q$ must be greater than $2 \pi \xi^{-1}$ or the quasiparticles can be created anywhere on the Fermi surface. EELS experiments which detect the gap in high-temperature superconductors have so far only been reported for near-zero momentum transfer [9]. 
The polarization $P_{b u l k}^{r e t}(\mathbf{Q}, \omega)$ in the superconducting state arises from two contributions. There is a "normal" polarization, where the vertex creates an electron and hole, which recombine at the other vertex. There is also an "anomalous" polarization, where the vertex creates an electron and hole, the electron turns into a hole through interaction with the gap, the hole turns into an electron, and then they recombine at the other vertex. The phase of the gap does not affect the normal process, but the anomalous process depends on the relative phase $\Delta \phi$ of the gap at the two points where quasiparticles are created. The sum of these two processes, at the threshold for electronic scattering processes, determines whether the discontinuity is its full size relative to the normal-state scattering probability at $T_{c}$. The ratio of the discontinuity $\Delta\left(d^{2} S / d \Omega d \omega\right)$ to the normal-state differential probability at $T_{c}$, $d^{2} S_{N} / d \Omega d \omega$, is

$$
\frac{\Delta\left(d^{2} S / d \Omega d \omega\right)}{d^{2} S_{N} / d \Omega d \omega}=\frac{\pi}{2} \cos ^{2}\left(\frac{\Delta \phi}{2}\right) .
$$

The full energy dependence at $T=0$ is

$$
\begin{aligned}
& \frac{d^{2} S(\omega) / d \Omega d \omega}{d^{2} S_{N}(\omega) / d \Omega d \omega}=\theta(\omega-\tilde{\Delta}(\mathbf{Q}))\left\{\cos ^{2}\left(\frac{\Delta \phi}{2}\right) E\left(\left[1-\left(\frac{\tilde{\Delta}(\mathbf{Q})}{\omega}\right)^{2}\right]^{1 / 2}\right)\right. \\
& \left.+\sin ^{2}\left(\frac{\Delta \phi}{2}\right)\left[\left(\frac{\omega+\tilde{\Delta}(\mathbf{Q})}{\omega}\right) E\left(\frac{\omega-\tilde{\Delta}(\mathbf{Q})}{\omega+\tilde{\Delta}(\mathbf{Q})}\right)-\frac{2 \tilde{\Delta}(\mathbf{Q})}{\omega} K\left(\frac{\omega-\tilde{\Delta}(\mathbf{Q})}{\omega+\tilde{\Delta}(\mathbf{Q})}\right)\right]\right\}
\end{aligned}
$$

where $E$ and $K$ are complete elliptic functions and $\theta$ is the Heavyside step function.

The normalized differential probability (Eq. (2)) is plotted in Fig. 2 for three values of $\Delta \phi$. In the isotropic superconductor, $\Delta \phi=0$ for all momenta transfers. For a $d_{x^{2}-y^{2}}$ gap 18,19, which is real but changes sign, $\Delta \phi$ can be $\pi$. An intermediate value of $\Delta \phi$ can only occur for a complex gap, which breaks time-reversal symmetry. Since the EELS experiments can be performed at very low substrate temperatures, the calculations presented here are for $T=0$.

Ref. [5] contains examples of the detailed temperature dependence of $\left(d^{2} S(T) / d \Omega d \omega\right)\left[d^{2} S_{N} / d \Omega d \omega\right]^{-1}$. This is the same quantity which is plotted there as the ratio of phonon linewidths at $T$ and $T_{c},\left(\gamma(T) / \gamma\left(T_{c}\right)\right)$. The above arguments concerning the 
structure of $\operatorname{Im} P_{b u l k}^{r e t}(\mathbf{Q}, \omega)$ are independent of the surface model which is now introduced to estimate the magnitude of $\left(d^{2} S\left(T_{c}\right) / d \Omega d \omega\right)$.

The scattering by superconducting electrons is through the polarization diagram — therefore the equations from dipole scattering theory [16] can be used. From Eq. (3.20) of Ref. [16], the differential probability for scattering into final angle $\Omega$ with energy loss $\hbar \omega$ is

$$
\frac{d^{2} S}{d \Omega d \omega}=\frac{2 e^{2} m^{2}|R|^{2}}{\pi \cos \theta_{s}}\left(\frac{k_{S}}{k_{I}}\right) \frac{P(\mathbf{Q}, \omega)}{\left[Q^{2}+\left(k_{I}^{z}-k_{S}^{z}\right)^{2}\right]^{2}}
$$

where $R$ is the reflection coefficient of the incident and scattered beam, $k_{S}$ is the momentum and $k_{S}^{z}$ is the $z$-momentum of the scattered beam, $k_{I}^{z}$ is the $z$-component of the momentum of the incident beam, $k_{s}^{z}=k_{s} \cos \theta_{s}, e$ and $m$ are the charge and mass of the electron, and

$$
P(\mathbf{Q}, \omega)=\int \mathrm{e}^{-i \omega t} d t \int_{-\infty}^{0} d z \int_{-\infty}^{0} d z^{\prime} \exp \left[Q\left(z+z^{\prime}\right)\right]\left\langle\rho\left(\mathbf{Q}, z^{\prime}, t\right) \rho(-\mathbf{Q}, z, 0)\right\rangle
$$

The small-angle condition is used twice to derive Eq. (3): (a) processes where the scattering interaction provides the momentum to reverse the sign of the electron's perpendicular momentum are ignored and (b) the electron beam is assumed not to penetrate substantially into the crystal compared to the range of influence of the density fluctuations on the electric field outside the crystal. Treating (a) correctly would provide a correction of order $Q^{2} /\left[\left(k_{I}^{z}-k_{S}^{z}\right)^{2}+Q^{2}\right]$. For the incident energies $(20 \mathrm{eV})$ of interest, and considering the smallest momentum transfer of interest $\left(\sim 0.4 \AA^{-1}\right)$, this correction is of order $2 \%$. Zone-boundarymomentum-transfer scattering, however, may have a substantial contribution from these corrections. (b) is not a concern because photoemission experiments [17] indicate an energetic electron could not penetrate more than one unit cell without being multiply scattered. Both assumptions (a) and (b) lead to an underestimate of the size of the effect. Certain effects which are difficult to model and which will decrease the actual size of the effect, such as multiple scattering and scattering from other excitations, will not be considered in this estimate.

We now discuss the structure of $P(\mathbf{Q}, \omega)$. In a material with a quasi-two-dimensional electronic structure, $P_{b u l k}^{r e t}(\mathbf{q}, \omega)$ is independent of $q_{z}$. For simplicity the surface is assumed to 
be a hard wall potential for the superconductor's electrons. Then $P(\mathbf{Q}, \omega)$ can be expressed in terms of $P_{b u l k}^{r e t}(\mathbf{Q}, \omega)$ as

$$
P(\mathbf{Q}, \omega)=\frac{2 e^{2}}{\pi Q}\left[\tan ^{-1}\left(\frac{Q}{q_{m}}\right)-\frac{q_{m} Q}{Q^{2}+q_{m}^{2}}\right] \operatorname{Im} P_{b u l k}^{r e t}(\mathbf{Q}, \omega),
$$

where $q_{m}$ is the zone-boundary momentum in the $z$-direction. The assumptions made about the surface influence the size of the effect through Eq. (5), but the proportionality to $P_{b u l k}^{r e t}(\mathbf{Q}, \omega)$ is independent of the surface model. The final form of Eq. (3) is

$$
\frac{d^{2} S}{d \Omega d \omega}=\frac{4 e^{4} m^{2}|R|^{2}}{\pi^{2} Q \cos \theta_{s}}\left(\frac{k_{S}}{k_{I}}\right)\left(\tan ^{-1}\left(\frac{Q}{q_{m}}\right)-\frac{q_{m} Q}{Q^{2}+q_{m}^{2}}\right) \frac{\operatorname{Im} P_{\text {bulk }}^{r e t}(\mathbf{Q}, \omega)}{\left[Q^{2}+\left(k_{I}^{z}-k_{S}^{z}\right)^{2}\right]^{2}} .
$$

For a momentum transfer of the inverse coherence length, and using the density of states information from photoemission [15], 5 states $/(\mathrm{eV} \mathrm{Cu}$ site $)$, the magnitude of the effect is $\sim 1 /($ steradian $\mathrm{eV})$.

This Communication concludes with the identification of specific momenta transfers of interest in $\mathrm{Bi}_{2} \mathrm{Sr}_{2} \mathrm{CaCu}_{2} \mathrm{O}_{8}$. The Fermi surface is shown in Fig. 1 [15]. The momentum transfer $0.5(\pi, \pi)$, whose quasiparticle channels are shown as vectors $1-3$, corresponds to the momentum of a phonon whose linewidth was tentatively identified as narrowing in Ref. [8]. The points on the Fermi surface probed by this momentum transfer are shown in one-eighth of the zone by the labeled filled triangles. Each of these thresholds may be independently observed if the gap is anisotropic. If the gap has $d_{x^{2}-y^{2}}$ symmetry 18,19, the momentum transfer $q_{n}$ should have an extremely low threshold loss. An additional check for $d_{x^{2}-y^{2}}$ symmetry is that the onset of a gap feature for all scattering momenta parallel to the $\Gamma-M$ direction should be sharp $(\Delta \phi=0)$. The onset for scattering momenta parallel to $\Gamma-X$ should be smooth and weak $(\Delta \phi=\pi)$.

I wish to acknowledge several illuminating discussions with C.M. Lieber and R.B. Phelps and the support of the U.S. Joint Services Electronics Program (JSEP) through ONR N00014-89-J-1023. 


\section{REFERENCES}

[1] W.N. Hardy, D.A. Bonn, D.C. Morgan, Ruixing Liang, and Kuan Zhang, Phys. Rev. Lett. 70, (1993) 3999.

[2] Z.-X. Shen, et al., Phys. Rev. Lett. 70, (1993) 1553.

[3] D.A. Wollman, D.J. Van Harlingen, W.C. Lee, D.M. Ginsberg, and A.J. Leggett, Phys. Rev. Lett. 71, (1993) 2134.

[4] M.E. Flatté, Phys. Rev. Lett. 70, (1993) 658.

[5] M.E. Flatté, S. Quinlan, and D.J. Scalapino, Phys. Rev. B 48, (1993) 10626.

[6] H. Chou, K. Yamada, J.D. Axe, S.M. Shapiro, G. Shirane, I. Tanaka, K. Yamane, and H. Kojima, Phys. Rev. B 42, (1990) 4272.

[7] N. Pyka, W. Reichardt, L. Pintschovius, G. Engel, J. Rossat-Mignod, and J.Y. Henry, Phys. Rev. Lett. 70, (1993) 1457.

[8] H.A. Mook, B.C. Chakoumakos, M. Mostoller, A.T. Boothroyd, and D. McK. Paul, Phys. Rev. Lett. 69, (1992) 2272.

[9] Y. Li, J. Liu, C.M. Lieber, Phys. Rev. Lett. 70, (1993) 3494. Y. Li, J.L. Huang, C.M. Lieber, Phys. Rev. Lett. 68, (1992) 3240. J.E. Demuth, B.N.J. Persson, F. Holtzberg, C.V. Chandrasekhar, Phys. Rev. Lett. 64, (1990) 603.

[10] Surface reconstruction and spin-flip scattering are ignored.

[11] V.M. Bobetic, Phys. Rev. 136, (1964) A1535.

[12] H.G. Schuster, Solid State Comm. 13, (1973) 1559.

[13] J.D. Axe and G. Shirane, Phys. Rev. Lett. 30, (1973) 214.

[14] S.M. Shapiro, G. Shirane, and J.D. Axe, Phys. Rev. B 12, (1975) 4899.

[15] D.S. Dessau, et al., Phys. Rev. Lett. 71, (1993) 2781. 
[16] D.L. Mills, Surf. Sci. 48, (1975) 59.

[17] Z.-X. Shen, private communication.

[18] N.E. Bickers, D.J. Scalapino, R.T. Scalettar, Int. J. Mod. Phys. B 1, (1987) 687; N.E. Bickers, D.J. Scalapino, S.R. White, Phys. Rev. Lett. 62, (1989) 961.

[19] P. Monthoux, A.V. Balatsky, D. Pines, Phys. Rev. Lett. 67, (1991) 3448; P. Monthoux, D. Pines, Phys. Rev. Lett. 69, (1992) 961. 


\section{FIGURES}

FIG. 1. Vectors $1-3$ are the non-degenerate ways to position the momentum $0.5(\pi, \pi)$ on the $\mathrm{Bi}_{2} \mathrm{Sr}_{2} \mathrm{CaCu}_{2} \mathrm{O}_{8}$ Fermi surface (dashed line) [15]. Observation of off-specular gap features with this momentum transfer would probe the gap at the points $1-3$ indicated by filled triangles. The onset of such features would need to be smooth and weak to be consistent with a $d_{x^{2}-y^{2}}$ gap symmetry. Any gap feature with momentum transfer $\overrightarrow{q_{n}}$ must occur at very low-frequency to be consistent

with $d_{x^{2}-y^{2}}$. An isotropic gap would have gap features in the scattering probability at energies similar to the on-specular feature [9].

FIG. 2. Scattering probability per unit energy and angle at $T=0$, normalized to the normal-state scattering probability. Solid line: relative phase $\Delta \phi=0$ between gaps at two places quasiparticles are created on the Fermi surface. Short dashed line: relative phase $\Delta \phi=\pi$. Long dashed line: relative phase $\Delta \phi=\pi / 2$. 